\title{
Computation of radiation from wire antennas on conducting bodies
}

\author{
Albertsen, N. Christian; Hansen, Jesper; Jensen, Niels E.
}

Published in:

I E E E Transactions on Antennas and Propagation

Publication date:

1974

Document Version

Publisher's PDF, also known as Version of record

Link back to DTU Orbit

Citation (APA):

Albertsen, N. C., Hansen, J., \& Jensen, N. E. (1974). Computation of radiation from wire antennas on conducting bodies. I E E E Transactions on Antennas and Propagation, 22(2), 200-206.

\section{General rights}

Copyright and moral rights for the publications made accessible in the public portal are retained by the authors and/or other copyright owners and it is a condition of accessing publications that users recognise and abide by the legal requirements associated with these rights.

- Users may download and print one copy of any publication from the public portal for the purpose of private study or research.

- You may not further distribute the material or use it for any profit-making activity or commercial gain

- You may freely distribute the URL identifying the publication in the public portal

If you believe that this document breaches copyright please contact us providing details, and we will remove access to the work immediately and investigate your claim. 


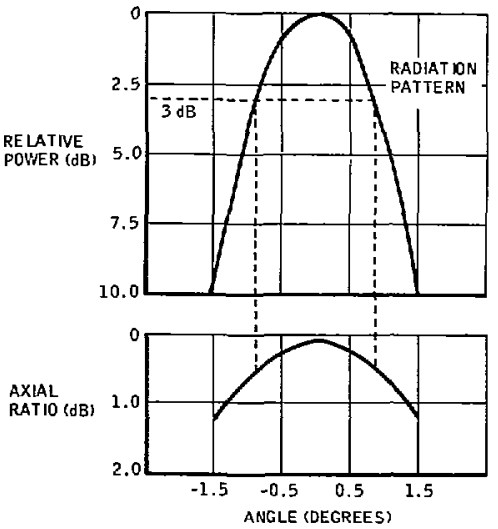

Fig. 10. Measured axial ratio of two-dimensional slot array.

the phase center separation in elevation between the linear arrays. Thus, two residual grating lobes remain. One is directly above and the other directly below the horizontal plane. As more pairs of linear arrays are added, the magnitude of these grating lobes is reduced until they disappear entirely. For the present case, in which there are only a total of eight linear arrays, the suppression of these residual grating lobes is incomplete. The degree of cancellation is also dependent on manufacturing tolerance of various components. Obviously, imbalance in either amplitude or phase between the linear arrays would produce a residual sidelobe. The reduction of this residual grating lobe is automatic when more linear arrays are employed, as in a typical two-dimensional array. An estimate of the residual grating lobe as a function of the number of linear arrays has been made. For a typical planar array antenna consisting of 40 linear arrays, the residual grating lobe is estimated to be below $40 \mathrm{~dB}$.

Radiation patterns were also measured for various scan angles, for various incident polarizations, and over a 6percent frequency band with comparable results.

The experimental array was set for circular polarization and the axial ratio was measured over various points within the main beam. As shown in Fig. 10, the axial ratio between the $3 \mathrm{~dB}$ points is within $0.5 \mathrm{~dB}$ and the cross polarization level was measured to be about $-30 \mathrm{~dB}$ at the main beam region and is generally much lower elsewhere.

\section{ACKNOWLEDGMENT}

The authors wish to thank Dr. R. Mack of AFCRL for helpful discussions and suggestions during the course of this program.

\section{REFERENCES}

[1] H. H. Hougardy and H. E. Shanks, "Arbitrarily polarized slot array," 1958 IRE WESCON Convention Record, Part I, Aug. 1958 , pp. $157-160$.

[2] R. S. Elliott, "Two-mode waveguide for equal mode velocities," IEEE Trans. Microwave Theory Techn., vol. MTF-16, pp. 282286, May 1968.

[3] P. Silvester, "A general high-order finite-element waveguide analysis program," IEEE Trans. Microwave Theory Tech., vol. MTT-17, pp. 204-210, Apr. 1969.

[4] J. S. Ajioka, "Two-mode waveguide slot array," U.S. Patent 3503073 , Mar. 24, 1970.

[5] P. D. Lacy and K. E. Miller, "An improved method for measuring. losses in short waveguide length," Hewlett-Packard $J$., vol. 9, Dec. 1957.

\title{
Computation of Radiation from Wire Antennas on Conducting Bodies
}

\author{
N. CHRISTIAN ALBERTSEN, JESPER E. HANSEN, AND NIELS E. JENSEN
}

\begin{abstract}
A theoretical formulation, in terms of combined magnetic and electric field integral equations, is presented for the class of electromagnetic problems in which one or more wire antennas are connected to a conducting body of arbitrary shape. The formulation is suitable for numerical computation provided that the overall dimensions of the structure are not large compared to the wave-
\end{abstract}

Manuscript received May 1, 1973; revised Oetober 1, 1973. This work was supported by the European Space Research and Technology Centre, Noordwijk, The Netherlands, under Contract 1340-71.

N. C. Albertsen and J. E. Hansen are with the Laboratory of Electromagnetic Theory, The Technical University of Denmark, Lyngby, Denmark.

N. E. Jensen was with the Laboratory of Electromagnetic Theory, The Technical University of Denmark, Lyngby, Denmark. He is now with the European Space Research and Technology Centre, Noordwijk, The Netherlands. length. A computer program is described, and test runs on various configurations involving a cylindrical body with one or more straight wires are presented. The results obtained agree well with experimental data.

\section{INTRODUCTION}

$\mathbf{F}$ IOR RADIATING systems that are not large compared to the wavelength, several methods are available for the determination of radiation patterns. For antennas mounted on structures which closely approximate simple geometrical shapes, the problem may well be attacked analytically [1]-[3], or by combined analytical and numerical methods [4]. However, when more complex 


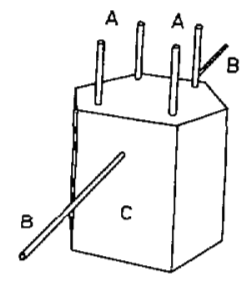

Fig. 1. Example of structure.

geometries are involved, as in the case of antennas on spacecrafts, resort has to be taken to purely numerical techniques, in which solutions to Maxwell's equations or integral equations derived from these, are obtained with the aid of a fast computer. A computer program for solving complicated radiation or scattering problems of the kind indicated is in many cases equally as accurate as experimental procedures, and more convenient. Various examples of such programs have been described in the past few years [5]-[10].

The present work deals with a specific class of radiating structures, the typical features of which are illustrated in Fig. 1. Here, a number of wire antennas $A$ and a number of booms (passive antennas) $B$ are attached to the surface of a metallic body $C$ which may, at least in principle, be arbitrarily shaped. The complete structure is assumed to have a maximum linear dimension which is not large compared to the wavelength. Fig. 1 is actually an idealization of the shape of a small scientific satellite with four antennas operating in a turnstile mode, and two booms for support of measurement equipment. Although this particular interpretation of Fig. 1 formed the primary motivation of the work reported here, the geometry is common to other types of radiating systems of interest, e.g., antennas on ships, cars, etc.

For the class of structures described it seems a natural choice to attempt a formulation in terms of integral equations. Several forms of integral equations suitable for solid bodies and thin wires exist [6]. Although the electric-field integral equation (EFIE) has been used as a basis for the numerical solution of scattering or antenna problems involving (large) bodies of revolution [11], the magnetic-field integral equation (MFIE) is more attractive for generally shaped voluminous structures. For thin wires or plates, however, the MFIE is known to fail, and one is forced to use the EFIE equation in such cases, or to modify the MFIE equation in a suitable way $[12],[13]$.

In view of the properties of the MFIE and the EFIE, the authors have used the MFIE for the solid body, whereas the EFIE in its thin-wire approximation has been applied to the wire parts of the structure. The mutual coupling between wires and body, and vice versa, is taken account of through the source terms in the integral equations [14]. A computer program based on this technique has been developed.

In Section II the important features of the MFIE and EFIE are summarized. In Section III it is explained how the coupling between body and wires may be computed using a surface current interpolation scheme. A formulation of the combined MFIE and EFIE equations particularly suitable for computer solution is given in Section IV. A short description of the structure of the computer program and examples of computations with experimental verifications are given in Section $V$.

\section{Magnetic and Electric Field Integrad EQUATIONS}

From Maxwell's equations, the following MFIE, for the surface current density $\bar{J}$ on the surface $S$ of a perfectly conducting body in an incident field with magnetic field strength $\bar{H}^{\text {ine }}$ may be derived [6]:

$$
\begin{aligned}
-\frac{1}{2} \bar{J}(\xi, \eta)+\hat{n} \times \int_{S}^{*} \bar{J}\left(\xi^{\prime}, \eta^{\prime}\right) & \times \nabla^{\prime} \phi d S^{\prime} \\
& =-\hat{n} \times \bar{H}^{\text {inc }}(\xi, \eta) .
\end{aligned}
$$

Here, $\hat{n}$ is an outward unit normal vector to the surface. Furthermore, $\phi=\exp (-j k R) / 4 \pi R$, where $R$ is the distance between the observation point $(\xi, \eta)$ and source point $\left(\xi^{\prime}, \eta^{\prime}\right)$ on area element $d S^{\prime}$, and where $k$ is the wavenumber. The asterisk denotes that the integration is carried out on the surface punctured at $(\xi, \eta)$. The symbol $\nabla^{\prime}$ denotes a gradient with respect to the primed coordinates. The time factor used is $\exp (j \omega t)$. In operator notation (2.1) may be written

$$
L_{M M} \bar{J}=-\hat{n} \times \bar{H}^{\text {inc }}
$$

For a perfectly conducting thin straight wire, the following EFIE may be derived [6]:

$$
\frac{1}{j \omega \epsilon} \int_{L}\left(\frac{\partial^{2} \phi}{\partial s^{2}}+k^{2} \phi\right) I\left(s^{\prime}\right) d s^{\prime}=-E_{s}^{\mathrm{inc}}(s)
$$

where it is assumed that the current $I$ has an axial component only (thin wire approximation). Here, $L$ denotes the interval for the coordinate $s^{\prime}$ of a source point on line element $d s^{\prime}$ along the wire, while

$$
\phi=\exp \left\{-j k\left[\left(s-s^{\prime}\right)^{2}+a^{2}\right]^{1 / 2}\right\} /\left[\left(s-s^{\prime}\right)^{2}+a^{2}\right]^{1 / 2}
$$

where $a$ is the wire radius. $E_{s}$ inc is the component of the electric field strength of the incident field in the direction of the wire, and $\epsilon$ the dielectric constant. In operator notation we have

$$
L_{E} I=-E_{s}^{\text {ine }} .
$$

Using pulse functions for expansion and point-matching, the moment method formulations [15] of (2.2) and (2.4) become, respectively

$$
\begin{array}{r}
{\left[\left\langle\delta_{p}, L_{M} W_{q}\right\rangle\right]\left[\bar{J}_{q}\right]=-\left[\left\langle\delta_{p}, \hat{n} \times \bar{H}^{\mathrm{inc}}\right\rangle\right],} \\
p=1,2, \cdots, q_{m} ; \quad q=1,2, \cdots, q_{m}
\end{array}
$$

and

$$
\begin{aligned}
& {\left[\left\langle\delta_{i}, L_{E} P_{j}\right\rangle\right]\left[Y_{j}\right]=-\left[\left\langle\delta_{i}, E_{s}^{\mathrm{inc}}\right\rangle\right]} \\
& \quad i=1,2, \cdots, j_{m} ; j=1,2, \cdots, j_{m} .
\end{aligned}
$$


In these formulas $\delta_{p}$ and $\delta_{i}$ are Dirac delta functions on $S$ and $L$, respectively. The centers of these (match points) are on the $p$ th of the $q_{m}$ patches into which the surface is divided, and on the $i$ th of the $j_{m}$ segments into which the wire is divided, respectively. $W_{q}$ and $P_{j}$ are pulse functions with amplitude unity on the $q$ th patch, and on the $j$ th segment, respectively, and zero elsewhere. Long-hand expansion of (2.6) may be found in [16].

While (2.5) is of a vector character, and may be split into two scalar systems of $q_{m}$ linear equations in the $2 q_{m}$ unknown components of the surface current density coefficients $\bar{J}_{q},(2.6)$ represents a system of $j_{m}$ linear equations in the $j_{m}$ unknown current coefficients $I_{j}$.

When implemented on a computer both the total number of surface patches $q_{m}$ at the MFIE and the total number of wire segments $j_{m}$ at the EFIE must be chosen large enough in order to obtain sufficiently accurate solutions $[6]$.

\section{Coupling Between Body ANd Wire}

In order to take the wire-body coupling into account, as described in Section I, we need formulas for the magnetic and electric fields from known current distributions. These may be found in standard texts, e.g., [17]. At a patch with unit normal vector $\hat{n}$ and a segment with unit tangential vector $\hat{s}$ we get the following contributions to the tangential magnetic and electric fields, respectively

$$
\hat{n} \times \bar{H}(I)=\hat{n} \times \int_{L} I\left(s^{\prime}\right) \hat{s}^{\prime} \times \nabla^{\prime} \phi d s^{\prime} \doteq L_{M}^{\prime} I
$$

and

$$
E_{s}(\bar{J})=\frac{1}{j \omega \epsilon} \hat{s} \cdot \int_{S} \nabla \times(\nabla \times(\bar{J} \phi)) d S \doteq L_{E}^{\prime} \bar{J}
$$

where we have introduced an operator notation along the same lines as above.

After having expanded the currents in (3.1) and (3.2) into pulse functions with coefficients $I_{j}$ and $\bar{J}_{q}$, in a similar manner as in Section II, we obtain the following matrix equation for the unknown currents in the mixed problem

$$
\begin{array}{r}
{\left[\begin{array}{c:c}
\left\langle\delta_{p}, L_{M} W_{q}\right\rangle & \left\langle\delta_{p}, L_{M}{ }^{\prime} P_{j}\right\rangle \\
\hline\left\langle\delta_{i}, L_{E}^{\prime} W_{q}\right\rangle & \left\langle\delta_{i}, L_{E} P_{j}\right\rangle
\end{array}\right]\left[\begin{array}{c}
\bar{J}_{q} \\
\hdashline I_{j}
\end{array}\right]} \\
=-\left[\frac{\left\langle\delta_{p}, \hat{n} \times \bar{H}^{\text {inc }}\right\rangle}{\hdashline\left\langle\delta_{i}, E_{s}^{\text {inc }}\right\rangle}\right]
\end{array}
$$

with $p=1,2, \cdots, q_{m} ; q=1,2, \cdots, q_{m} ; i=1,2, \cdots, j_{m} ;$ and $j=1,2, \cdots, j_{m}$.

This equation is of mixed scalar and vector form, and may be expanded into scalar form alone by introducing the proper decompositions of the vector quantities.

In order to compute the off-diagonal (coupling) coefficients in (3.3) a careful treatment of the currents close to the wire attachment area is necessary. The operator $L_{M}{ }^{\prime}$ is well behaved, hence it suffices to use pulse functions in (3.1) for all segments including the attachment segment.

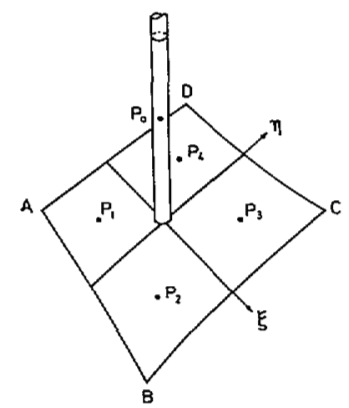

Fig. 2. Details of wire attachment area.

The operator $L_{E}^{\prime}$, on the other hand, has a singularity of third order, which makes it necessary to use at patches on which wires are attached, a more accurate expression for the surface current density $\bar{J}$ than pulse functions. The technique used is described below for the case of quadrilateral patches.

In Fig. 2, $A B C D$ is a quadrilateral patch in a body segmentation scheme set up before the wires are brought into the picture. In order to introduce an interpolation of the surface current density on $A B C D$ which takes into account the attachment of a wire, $A B C D$ is divided into four patches as shown. A local coordinate system $(\xi, \eta)$ is introduced in which $A, B, C$, and $D$ have the coordinates $\left(\xi_{1}, \eta_{1}\right),\left(\xi_{2}, \eta_{2}\right),\left(\xi_{3}, \eta_{3}\right)$, and $\left(\xi_{4}, \eta_{4}\right)$, respectively. The match points of the four patches are denoted $P_{1}, \cdots, P_{4}$, and the unknown surface eurrent densities in these points $\bar{J}_{1}, \cdots, \bar{J}_{4}$. The match point on the first wire segment is $P_{0}$ and the corresponding unknown current $I_{0}$. The coordinates for $P_{1}, \cdots, P_{4}$ are $\left(\xi_{1} / 2, \eta_{1} / 2\right), \cdots,\left(\xi_{4} / 2, \eta_{4} / 2\right)$, respectively.

A suitable expansion of the surface current density $\bar{J}(\xi, \eta)$ over $A B C D$ should meet the following requirements:

$$
\bar{J}\left(\xi_{i} / 2, \eta_{i} / 2\right)=\bar{J}_{i}, \quad i=1, \cdots, 4
$$

and

$$
\nabla_{s} \cdot \bar{J}(\xi, \eta)=J_{0}(\xi, \eta)-I_{0} \delta(\xi, \eta)
$$

where $\nabla_{s} \cdot$ denotes surface divergence, and where $J_{0}(\xi, \eta)$ is continuous across $A B C D$. One such expansion is

$$
\bar{J}(\xi, \eta)=I_{0} \frac{\xi \hat{\xi}+\eta \hat{\eta}}{2 \pi\left(\xi^{2}+\eta^{2}\right)}+\sum_{i=1}^{4} \bar{J}_{i}^{\prime} g_{i}(\xi, \eta) .
$$

The interpolation functions, $g_{1}(\xi, \eta), \cdots, g_{4}(\xi, \eta)$, are required to have the following properties across $A B C D: 1$ ) $g_{i}(\xi, \eta)$ is differentiable; 2) $g_{i}\left(\xi_{j}, \eta_{j}\right)=\delta_{i j}$, for $i=1, \cdots, 4$ and $j=1, \cdots, 4$, where $\delta_{i j}$ is the Kronecker delta; and 3) $g_{1}(\xi, \eta)+\cdots+g_{4}(\xi, \eta)=1$.

These requirements are fulfilled by the hyperbolic paraboloids used in this study. Hyperbolic paraboloids are particularly well suited for quadrilateral surface patches, and are merely specific examples of the more general "wedge functions" introduced by Wachspress [18] as interpolation functions for surface patches of a general shape.

The fields due to the currents on the attachment patches and segment may be expressed in terms of $\bar{J}_{1}^{\prime}, \cdots, \bar{J}_{4}^{\prime}$, 
and $I_{0}$ as the moments

$$
\begin{aligned}
{\left[\left\langle\delta_{p}, \hat{n} \times \bar{H}\right\rangle\right] } & =[B]\left[\bar{J}^{\prime}\right]+\left[W_{B}\right] I_{0} \\
\left\langle\delta_{0}, E_{s}\right\rangle & =\left[B_{W}\right]\left[\bar{J}^{\prime}\right]+W I_{0}
\end{aligned}
$$

where $[B]$ is a matrix derived from $(2.5), W$ is a coefficicnt derived from (2.6) and $\left[W_{B}\right]$ and $\left[B_{W}\right]$ are matrices derived as inner products between (3.1) and $\delta_{p}$, and between (3.2) and $\hat{\delta}_{0}$, respectively. Here $\delta_{p}$ and $\delta_{0}$ are delta functions defined in Section II. In the computations $[B]$, $\left[W_{B}\right]$, and $W$ are evaluated using the simple technique used for all other elements of (3.3), i.e., the unknown current densities are approximated by pulse functions, while $\left[B_{W}\right]$ is computed as the moments of $\delta_{0}$ (at $P_{0}$ ) and (3.2) with (3.6) inserted.

If (3.6) is inserted into (3.4), a set of linear equations is obtained which may be solved for $\tilde{J}_{1}{ }^{\prime}, \cdots, J_{4}{ }^{\prime}$. In this manner the fields at $P_{0}, P_{1}, \cdots, P_{4}$ due to currents on the attachment patches and segment are expressed in terms of the unknowns $\bar{J}_{1}, \cdots, \bar{J}_{4}$ and $I_{0}$ occurring in (3.3).

It is noted that the interpolation technique described permits continuity of the current at the attachment point of the wire without introduction of new unknown quantities.

\section{Formulation for Computer Solution}

In this section we shall show how (3.3) may be rewritten in order to cut down computer storage requirements. From (3.3) we have by multiplying out formally

$$
\begin{array}{r}
{\left[\left\langle\delta_{p}, L_{M} W_{q}\right\rangle\right]\left[\bar{J}_{q}\right]+\left[\left\langle\delta_{p}, L_{M}{ }^{\prime} P_{j}\right\rangle\right]\left[I_{j}\right]} \\
=-\left[\left\langle\delta_{p}, \hat{n} \times \bar{H}^{\mathrm{inc}}\right\rangle\right]
\end{array}
$$

and

$$
\begin{array}{r}
{\left[\left\langle\delta_{i}, L_{E}^{\prime} W_{q}\right\rangle\right]\left[\bar{J}_{q}\right]+\left[\left\langle\delta_{i}, L_{E} P_{j}\right\rangle\right]\left[I_{j}\right]} \\
=-\left[\left\langle\delta_{i}, E_{s}{ }^{i n c}\right\rangle\right] .
\end{array}
$$

Equation (4.1) may now be solved for $\left[\bar{J}_{q}\right]$.

$$
\begin{aligned}
{\left[\bar{J}_{q}\right]=} & -\left[\left\langle\delta_{p}, L_{M} W_{q}\right\rangle\right]^{-\mathrm{1}}\left[\left\langle\delta_{p}, \hat{n} \times \bar{H}^{\mathrm{inc}}\right\rangle\right] \\
& -\left[\left\langle\delta_{p}, L_{M} W_{q}\right\rangle\right]^{-\mathrm{t}}\left[\left\langle\delta_{p}, L_{M} P_{j}\right\rangle\right]\left[I_{j}\right]
\end{aligned}
$$

which by insertion in (4.2) yields

$$
\begin{aligned}
(- & {\left[\left\langle\delta_{i}, L_{E}^{\prime} W_{q}\right\rangle\right]\left[\left\langle\delta_{p}, L_{M} W_{q}\right\rangle\right]^{-1}\left[\left\langle\delta_{p}, L_{M}{ }^{\prime} P_{j}\right\rangle\right] } \\
& \left.+\left[\left\langle\delta_{i}, L_{E} P_{j}\right\rangle\right]\right)\left[I_{j}\right] \\
= & {\left[\left\langle\delta_{i}, L_{E}^{\prime} W_{q}\right\rangle\right]\left[\left\langle\delta_{p}, L_{M} W_{q}\right\rangle\right]^{-1}\left[\left\langle\delta_{p}, \hat{n} \times \bar{H}^{\text {inc }}\right\rangle\right] } \\
& -\left[\left\langle\delta_{i}, E_{s}^{\text {inc }}\right\rangle\right] .
\end{aligned}
$$

When this equation has been solved for $\left[I_{j}\right],\left[\bar{J}_{q}\right]$ may be found from (4.3).

Equation (4.4) is of a form similar to the moment solution (2.6) of the EFIE for a wire in free space. However, extra terms occur on both sides of the equality sign. These precisely account for the presence of the conducting body, and (4.4) may be considered to be the moment method formulation of an electric field integral equation similar to (2.3), with the (free space) Green's function $\phi$ modified to take the conducting body into account. This is analogous to the integral equation formulation given in [4] for the particular case of a monopole on a sphere, where the modified Green's function is available in analytical form.

An equation for $\left[\bar{J}_{q}\right]$ analogous to (4.4) may also be derived. The result is

$$
\begin{aligned}
([ & \left.\left\langle\delta_{p}, L_{M}{ }^{\prime} P_{j}\right\rangle\right]\left[\left\langle\delta_{i}, L_{E} P_{j}\right\rangle\right]^{-1}\left[\left\langle\delta_{i}, L_{E}^{\prime} W_{q}\right\rangle\right] \\
& \left.\quad+\left[\left\langle\delta_{p}, L_{M} W_{q}\right\rangle\right]\right)\left[\bar{J}_{q}\right] \\
= & {\left[\left\langle\delta_{p}, L_{M} P_{j}\right\rangle\right]\left[\left\langle\delta_{i}, L_{E} P_{j}\right\rangle\right]^{-1} \quad\left[\left\langle\delta_{i}, E_{s}{ }^{\text {inc }}\right\rangle\right] } \\
& -\left[\left\langle\delta_{p}, \hat{n} \times \bar{H}^{\mathrm{inc}}\right\rangle\right] .
\end{aligned}
$$

This equation may be considered to be the moment method formulation of an MFIE similar to (2.1), with a kernel that takes the conducting wire into account.

For a problem involving $j_{m}$ segments and $q_{m}$ patches the original matrix in (3.3) has $\left(2 q_{m}+j_{m}\right)^{2}$ elements. With the preceding approach totally no more than two matrices with a total number of elements not exceeding $2 q_{m}\left(2 q_{m}+j_{m}\right)$ or $j_{m}\left(j_{m}+2 q_{m}\right)$, any of which is the largest figure, must be in core storage in the computer, simultaneously.

In the present work, (4.4) forms the basis of computations. In the computer program, the operators $L_{M}$ and $L_{E}$ have been evaluated using rectangular rule for the integration. The coupling between the attachment segment and the attachment patch is computed using Romberg integration in $L_{M z^{\prime}}$ and a rectangular rule with 100 subdivisions in $L_{E}{ }^{\prime}$. All other couplings are computed using a simple rectangular rule in both $L_{M^{\prime}}$ and $L_{E}{ }^{\prime}$.

The computation of the radiation pattern from $\left[I_{j}\right]$ and $\left[\bar{J}_{q}\right]$ is a standard procedure, which may be found in textbooks, e.g., [17]. For this reason details are omitted.

\section{Computations and Comparisons with Experiments}

The computer program constructed for the present investigation consists of a main program and 34 Fortran subroutines. The sequence of computations is divided into four distinct parts.

First, the complete geometry is specified. This includes all coordinates of the structure as well as the segmentation parameters for body, antennas, and passive booms. In the second part of the computer program, the left-hand side matrix in (4.4) is formed and stored in an array. In the third part of the program, (4.4) is solved for the currents on all antennas and other wires attached to the body. Equation (4.3) then yields the surface current densities. Finally, the radiation pattern is computed. A number of test runs of the program and comparisons with experiments have been made for a circular cylindrical body with straight antennas and passive booms.

The experimental model is shown in Fig. 3 . The height and diameter of the cylindrical body are $220 \mathrm{~mm}$ and 200 $\mathrm{mm}$, respectively. Inside the model, a battery-powered transmitter for the frequency range $0.5-1.0 \mathrm{GHz}$ is placed. On the surface of the model, monopole antennas and passive booms may be mounted in a variety of positions. 


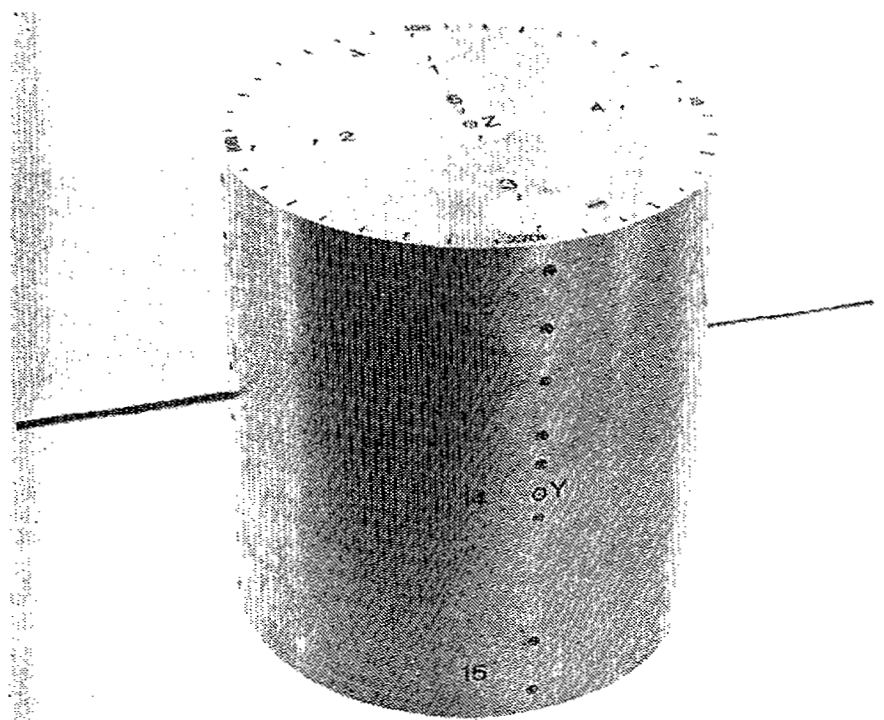

Fig. 3. Cylindrical model used for experiments.

(a)

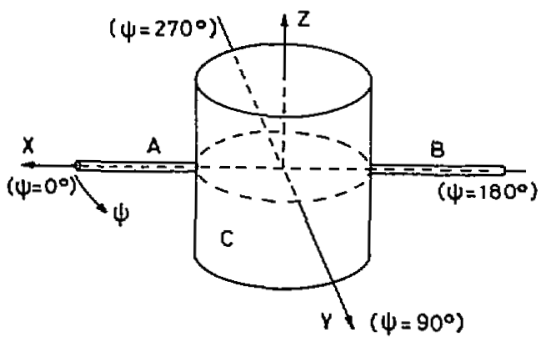

(b)

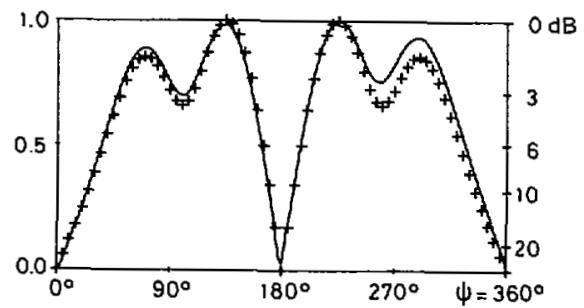

(c)

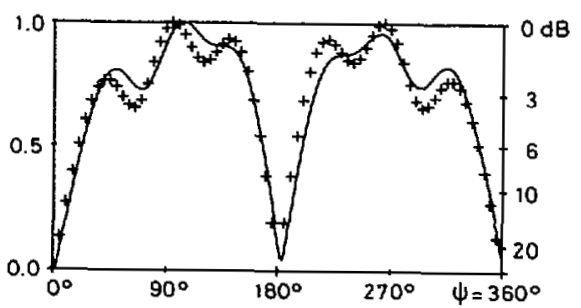

(d)

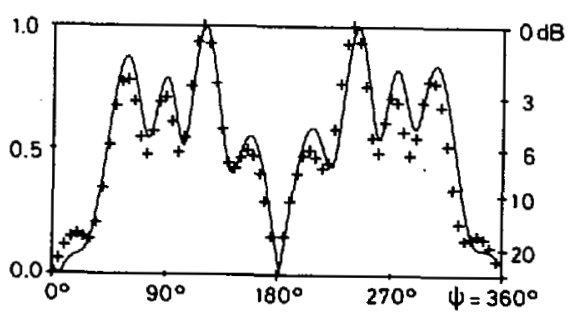

Fig. 4. $x y$-polarized radiation patterns in the $x y$-plane (field strength, normalized to maximum value of unity, as function of angle $\psi$ ) for monopole antenna $(A)$ on metallic cylinder $(C)$ with and without straight boom $(B)$. Cylinder height $=0.22 \mathrm{~m}$, cylinder diameter $=0.20 \mathrm{~m}$, wavelength $=0.36 \mathrm{~m}$. (a) Configuration, $x y z$-coordinate system (centered at cylinder midpoint), and definition of angular variable $\psi$. (b) Radiation pattern without boom; monopole length $=0.12 \mathrm{~m}$. (c) Radiation pattern for boom length of $0.09 \mathrm{~m}$; monopole length $=0.08 \mathrm{~m}$. (d) Radiation pattern for boom length of $0.44 \mathrm{~m}$; monopole length $=0.08 \mathrm{~m}$.

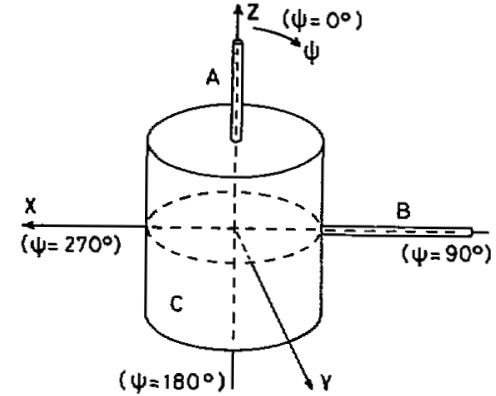

(a)

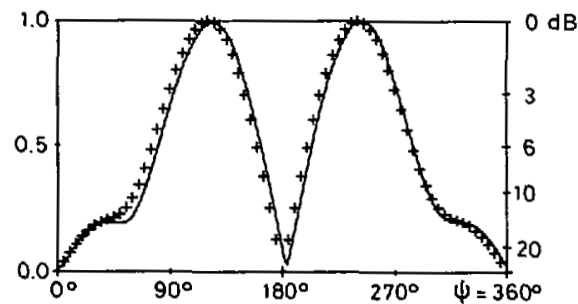

(b)

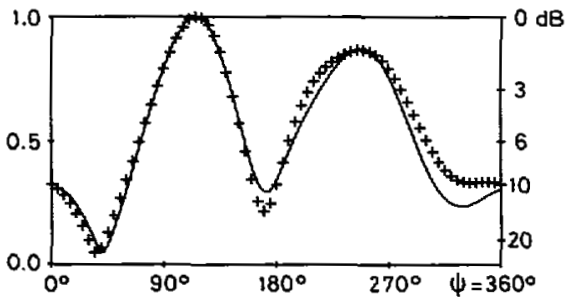

(c)

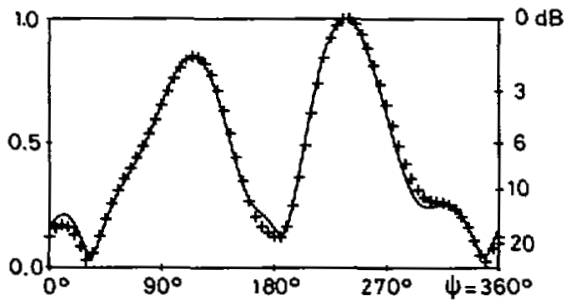

(d)

Fig. 5. $z x$-polarized patterns in the $z x$ plane (field strength, normalized to maximum value of unity, as function of the angle $\psi$ ) for monopole antenna $(A)$ on a metallic cylinder $(C)$ with and without a straight boom $(B)$. Cylinder height $=0.22 \mathrm{~m}$, cylinder diameter $=0.20 \mathrm{~m}$, monopole length $=0.12 \mathrm{~m}$; wavelength $=$ $0.48 \mathrm{~m}$. (a) Configuration, $x y z$-coordinate system (centered at cylinder midpoint), and definition of angular variable $\psi$. (b) Radiation pattern without boom. (c) Radiation pattern for boom length of $0.18 \mathrm{~m}$. (d) Radiation pattern for boom length of $0.44 \mathrm{~m}$.

The antenna is $2 \mathrm{~mm}$ in diameter while booms (in metallic connection with the body) are $4 \mathrm{~mm}$ in diameter.

During the measurements the received signals were recorded digitally on paper tape. These tapes were used later as input data for a plotting program. Measured patterns (solid line) for a number of representative configurations are shown together with the corresponding computer radiation patterns (crosses) in Figs. 4-6.

The small unsymmetries in the measured patterns in Figs. 4 and 6, where the configurations have complete symmetry with respect to the $x z$ plane, give an impression of the role of the measurement inaccuracies. These were due to wall reflections in the Radio Anechoic Room where the patterns were taken.

In Fig. 4 the influence on the radiation pattern of 
TABLE I

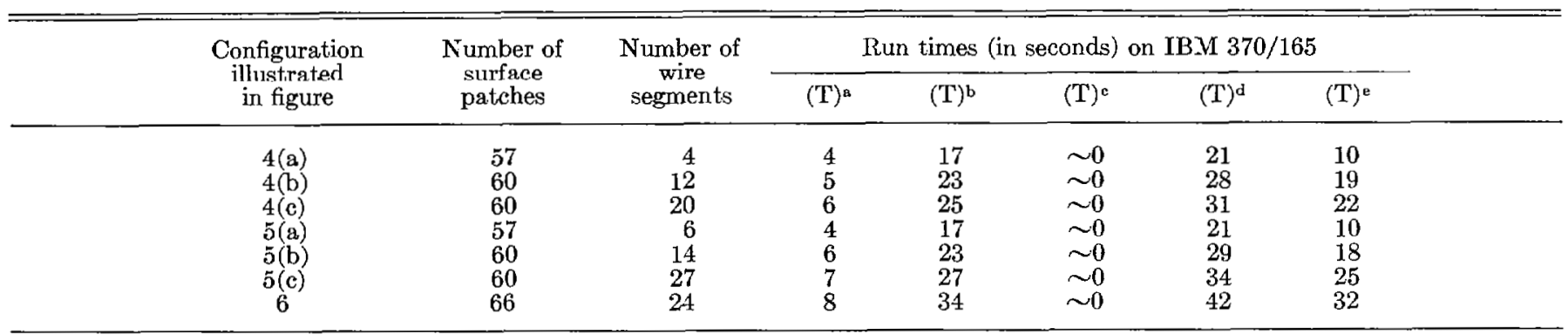

a Computation of $L_{M}$ and $L_{M}{ }^{\prime}$ for (4.1).

b Solution of (4.1).

- Computation of $L_{E}$ and $L_{E}{ }^{\prime}$ and solution of $(4,4)$.

d Total time for computation of current distributions.

e Additional time for computation of radiation patterns.

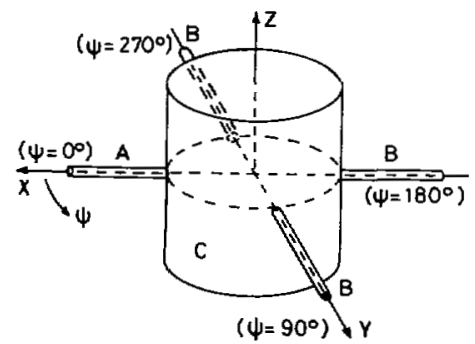

(a)

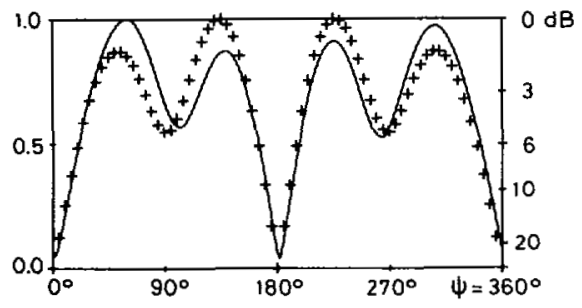

(b)

Fig. 6. $x y$-polarized radiation pattern in $x y$ plane (field strength, normalized to maximum value of unity, as function of the angle 4) for a monopole antenna $(A)$ on metallic cylinder $(C)$ with four straight booms $(B)$. Cylinder height $=0.22 \mathrm{~m}$, cylinder diameter $=0.20 \mathrm{~m}$, monopole length $=0.12 \mathrm{~m}$, boom length $=$ $0.13 \mathrm{~m}$, wavelength $=0.58 \mathrm{~m}$. (a) Configuration, $x y z$-coordinate system (centered at cylinder midpoint), and definition of angular variable $\psi$. (b) Radiation pattern.

adding booms diametrically opposite to an antenna on the curved surface of the cylinder, is shown The undisturbed pattern is shown in Fig. 4 (a), while patterns for booms of lengths $\lambda / 4$ and $5 \lambda / 4$ are given in Figs. $4(b)$ and (c), respectively. It is seen that the radiation pattern in the $x y$ plane is changed noticeably by the addition of booms. In all three cases the computations predict the measured behavior satisfactorily.

For the examples in Fig. 5 the antenna is placed at the top of the cylinder. The undisturbed pattern is shown in Fig. 5(a), while patterns for booms of lengths $\lambda / 4$ and $3 \lambda / 4$ are given in Figs. 5 (b) and (c), respectively. Also in these cases the measured results are satisfactorily predicted by the computations.

Fig. 6 demonstrates the numerical prediction of the radiation pattern of an antenna on a cylinder with three quarterwave booms.

No studies of the relative numerical convergence as a function of sampling density were made. The segmentation

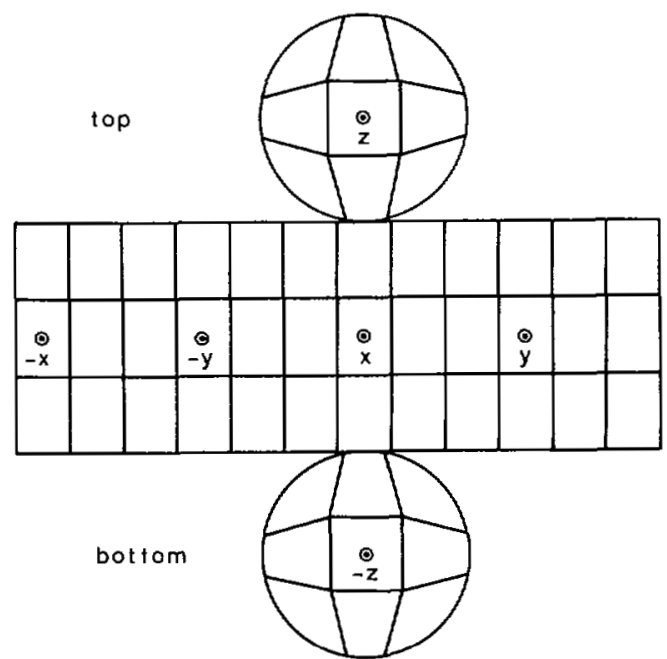

Fig. 7. Segmentation of cylinder.

of body and wires were made with as many patches and segments as permitted by the available core storage in the computer (500k bytes) and the computations were verified by comparison with experiments. In all cases the number of surface patches was larger than the number used in [6] in an MFIE computation of surface currents on a sphere in a plane wave field.

The number of surface patches, wire segments, and run times for the seven examples described above were as given in Table I.

Other computational examples and a more detailed description of the computer program may be found in $[19]$. The location of booms and antennas in the cylinder version of the program is restricted to surface patches of quadrilateral shape. No boom or antenna should be placed directly on the edge of the cylinder or in the triangular shaped patches on the end surfaces of the cylinder shown in Fig. 7. No special treatment of the cylinder edges were attempted. Apparently, this is not of any practical importance for the computations, although the MFIE obviously is not valid for field points right on the edge.

\section{Conclusion}

A theoretical formulation in terms of combined magnetic and electric field integral equations has been presented for 
antenna and scattering problems involving wires connected to a conducting body. Numerical computations using the method of moments for the solution have been made for a configuration consisting of a cylindrical body with straight wires attached to the surface. Comparison between the computations and experiments shows good agreement.

The formulation and computer program described in this paper seem suitable for solving electromagnetic problems in which thin wires are connected to a conducting body. Although the primary motivation for the work presented was the need for computerized prediction of radiation patterns for spacecraft VHF-monopole antennas, the technique may prove useful also for the examination of radiation from antennas on small aircrafts, portable radio sets, etc.

\section{ACKNoWLEDGMENT}

The authors wish to thank G. Paci of the European Space Research Organisation for suggesting the present investigation. The numerical computations were made at the NEUCC computing center at the Technical University of Denmark.

\section{REFERENCES}

[1] F. V. Schultz, "The scattering of electromagnetic waves by perfectly reflecting objeets of complex shape," School Elec. Eng., Purdue Univ., Indiana, Rep. TR-EE68-39, 1968.

[2] D. M. Bolle and M. D. Morganstern, "Monopole and conic antennas on spherical vehicles," IEEE Trans. Antennas Propagat., vol. AP-17, pp. 477-84, July 1969 .

[3] P. Bruscaglioni and A. Consortini, "Irraggiamento di un monopolo su una sfera conduttrice," Alta Freq., vol. 36, pp. $224-26,1967$.

[4] F. M. Tesche and A. R. Neureuther, "Radiation patterns for two monopoles on a perfectly conducting sphere," IEEE Trans. Antennas Propagat., vol. AP-18, pp. 692-94, Sept. 1970.
[5] E. K. Miller et al., "Numerical analysis of aircraft antennas," in Proc. Conf. Environmental Effects on Antenna Performance, $\mathrm{pp}$. $55-58,1969$.

[6] A. J. Poggio and E. K. Miller, "Integral equation solutions of three-dimensional scattering problems," in Computer Techniques for Electromagnetics, R. Mittra, Ed. Oxford: Pergamon, 1973, ch. 4.

[7] P. L. E. Uslenghi, "Computation of surface currents on bodies of revolution," Alta Freq., vol. 39, pp. 213E-224E, Aug. 1970.

[8] G. A. Thiele and M. Travieso-Diaz, "Radiation of a monopole antenna on the base of a conical structure," in Proc. Conf. Environmental Effects on Antenna Performance, pp. 99-102, 1969.

[9] C. L. Bennett, A. M. Auckenthaler, and J. D. DeLorenzo, "Transient scattering by three-dimensional conducting surfaces with wires," in 1971 G-AP Int. Symp. Dig., pp. 349-351, Sept. 1971.

[10] J. E. Jones, J. H. Richmond, and T. G. Campbell, "A numerical method for obtaining radiation patterns of space shuttle annular slot antennas," 1972 G-AP Int. Symp. Dig., pp. 189-192, Dec. 1972.

[11] J. R. Mautz and R. F. Harrington, "Radiation and scattering from bodies of revolution," Appl. Sci. Res., vol. 20, pp. 405435, June 1969.

[12] R. Mittra, "How to use the $H$-integral equation for electrically thin antennas and scatterers," in 1972 USNC-URSI Spring Meeting Abst., 1972.

[13] R. Mittra, W. A. Davis, and D. V. Jamnejad, "An integral equation for plane wave scattering by thin plates," in 1972 USNC-URSI Fall Meeting Abst., pp. 93-94, Dec. 1972.

[14] N. C. Albertsen, J. E. Hansen, and $\mathrm{N}$. E. Jensen, "Numerical prediction of radiation patterns for antennas mounted on spacecrafts," Conference on Aerospace Antennas, IEE Conference Publication Number 77, London, pp. 219-228, June 1971.

[15] R. F. Harrington, Field Computation by Moment Methods. New York: Macmillan, 1968.

[16] J. H. Richmond, "A wire-grid model for scattering by conducting bodies," IEEE Trans. Antennas Propagat., vol. AP-14, pp. 782-86, Nov. 1966.

[17] R. E. Collin and F. J. Zucker, Antenna Theory. New York: McGraw-Hill, 1969, pt. I, ch. 2.

[18] E. L. Wachspress, "A rational basis for function approximation," in Publ. Conf. Applications of Numerical Analysis. Berlin, Germany: Springer-Verlag, 1971.

[19] N. C. Albertsen, J. E. Hansen, and N. E. Jensen, "Computation of spacecraft antenna radiation patterns," Lab. Electromag. Theory, Tech. Unir. Denmark, Lyngby, Denmark. Rep. R 108, June 1972 ; also Rep. ESRO CR-20', Sept. 1973. 